TRANSACTIONS OF THE

AMERICAN MATHEMATICAL SOCIETY

Volume 357, Number 12, Pages 4885-4896

S 0002-9947(05)03887-0

Article electronically published on July 19, 2005

\title{
EXTENDED HARDY-LITTLEWOOD INEQUALITIES AND SOME APPLICATIONS
}

\author{
HICHEM HAJAIEJ
}

Dedicated to my Mother: To you Omi

\begin{abstract}
We establish conditions under which the extended Hardy-Littlewood inequality

$$
\int_{\mathbb{R}^{N}} H\left(|x|, u_{1}(x), \ldots, u_{m}(x)\right) d x \leq \int_{\mathbb{R}^{N}} H\left(|x|, u_{1}^{*}(x), \ldots, u_{m}^{*}(x)\right) d x,
$$

where each $u_{i}$ is non-negative and $u_{i}^{*}$ denotes its Schwarz symmetrization, holds. We also determine appropriate monotonicity assumptions on $H$ such that equality occurs in the above inequality if and only if each $u_{i}$ is Schwarz symmetric. We end this paper with some applications of our results in the calculus of variations and partial differential equations.
\end{abstract}

\section{INTRODUCTION}

The most novel part of this paper is the characterization of cases of equality in the extended Hardy-Littlewood inequalities. Our approach also enables us to prove

$$
\int_{\mathbb{R}^{N}} H\left(|x|, u_{1}(x), \ldots, u_{m}(x)\right) d x \leq \int_{\mathbb{R}^{N}} H\left(|x|, u_{1}^{*}(x), \ldots, u_{m}^{*}(x)\right) d x
$$

in a simple and original way, recovering the main result of [5]; let us point out at this early stage that there is no optimal result, including the one of this paper, concerning (1.1).

For dealing with (1.1) (and cases of equality in (1.1)) in the calculus of variations and in some other domains, it is fundamental to establish it for integrands $H$ which are not necessarily continuous with respect to the distance $|x|$ (see the introduction of 3 for more details). In Theorem 4.2 of [2], we proved (1.1) under minimal regularity assumptions when $m=2$; our approach set out in [2] still applies to $m>2$, thus we can easily extend Proposition 4.1 of [2] (and consequently Theorem 4.2). However, this method uses approximation arguments in such a way that it seems impossible to read off cases of equality in (1.1). In case $H \equiv G$ is absolutely continous with respect to the second variable, we developed in [1] a self-contained method thanks to which we determined optimal monotonicity

Received by the editors January 13, 2004.

2000 Mathematics Subject Classification. Primary 26D15.

(C)2005 American Mathematical Society
ublic domain 28 years from publication Reverts to public domain 28 years from publication 
assumptions under which

$$
\int_{\mathbb{R}^{N}} G(|x|, u(x)) d x=\int_{\mathbb{R}^{N}} G\left(|x|, u^{*}(x)\right) d x
$$

if and only if $u=u^{*}$ a.e.

Moreover, under minor modifications, this method still applies to establish cases of equality in (1.1) when, except for one $u_{i}$, the other functions are already radially decreasing, but it cannot be applied directly to determine cases of equality in (1.1) when $m>1$ and each $u_{i}$ is arbitrary. This case is the most interesting.

In this paper, we present two approaches to solve this problem:

- The first one consists of reducing the study of cases of equality in (1.1) to the one of integrands which are products of "derivatives" of $H$ and step functions depending only on one $u_{i}$ (see (3.9) and (3.10) in the proof of Theorem 3.1). We then obtain $m$ equations having the form studied in Lemma 3.6 of [1, and we can conclude using this result.

- It also turns out that the introduction of an appropriate intermediate step, enabling us to reduce the treatment of cases of equality in (1.1) to $m$ equations such that each equation has the form (1.2) for each $u_{i}$ (see (3.17) and (3.18) in the proof of Theorem 3.3), is fruitful. This work is completed in Theorem 3.3 in the case $m=2$ and can be extended to $m>2$.

Let us remark that classes of functions given in Theorem 3.1 and Theorem 3.3 are different (see Example 3.2). Together, they include a large class of functions arising in mathematical physics and economics. Note also that a "subtle" combination of our first approach and the second one gives us other classes of functions $H$ for which (1.1) holds with equality if and only if each $u_{i}$ is Schwarz symmetric (see Remark 3.6).

Proofs of results in Section 3 are the most "tricky", if not the most novel part of this paper. Despite the numerous applications of such results, we are not aware of any previous papers dealing with the establishment of cases of equality in (1.1) (apart from [1).

We end this paper with some applications of our results in the calculus of variations and partial differential equations (see Theorem 4.1 and Theorem 4.2).

\section{Notation, DEFinitions AND PRELIMINARIES}

All statements about measurability refer to the Lebesgue measure, mes, on $\mathbb{R}^{N}$ or $[0, \infty)$. For $r \geq 0, B(0, r)=\left\{x \in \mathbb{R}^{N}|| x \mid<r\right\}$. There is a constant $V_{N}>0$ such that $B(0, r)=V_{N} r^{N}$ for all $r>0$.

For a measurable subset $A$ of $\mathbb{R}^{N}$ with finite measure, $A^{*}=B(0, r)$, where $V_{N} r^{N}=\operatorname{mes} A$. The characteristic function of a subset $A$ of $\mathbb{R}^{N}$ is denoted by $1_{A}$. Let $M_{N}$ denote the set of all extended real-valued functions which are measurable on $\mathbb{R}^{N}$. For $u \in M_{N}$ and $t \in \mathbb{R}$, let $d_{u}(t)=\operatorname{mes}\left\{x \in \mathbb{R}^{N} \mid u(x)>t\right\}$ be its distribution function and set

$$
F_{N}=\left\{u \in M_{N} \mid 0 \leq u<\infty \text { a.e. on } \mathbb{R}^{N} \text { and } d_{u}(t)<\infty \text { for all } t>0\right\},
$$

the set of Schwarz symmetrizable functions and $F_{N}^{+}=\left\{u \in F_{N} \mid u>0\right\}$. For $u \in F_{N}$, its Schwarz symmetrization, denoted by $u^{*}$, is the unique function such that $d_{u^{*}}(t)=d_{u}(t)$ for every $t>0$ and $u^{*}(x)=h(|x|)$, where $h:(0, \infty) \longrightarrow[0, \infty)$ 
is a non-increasing right-continous function. We say that an element $u \in F_{N}$ is Schwarz symmetric if $u=u^{*}$ a.e.

Simple functions can be symmetrized in a very simple way. Let

$$
E_{N}=\left\{u \in F_{N} \mid u \text { is a simple function }\right\} .
$$

That is to say, $E_{N}$ is the set of all functions which can be written as

$$
u=\sum_{i=0}^{k} a_{i} 1_{A_{i}} \quad \text { for some } k \in \mathbb{N},
$$

where $a_{i} \in(0, \infty)$ with $a_{i}>a_{i+1}, A_{i}$ are measurable subsets of $\mathbb{R}^{N}$ with mes $A_{i}<$ $\infty$ and $A_{i} \cap A_{j}=\emptyset$ for $i \neq j$.

In [2], we proved that any element $u \in E_{N}$ can be rewritten as

$$
u=\sum_{i=0}^{k} h_{i} u_{i}
$$

where

$$
\begin{aligned}
& h_{i}=a_{i}-a_{i+1}, \quad \text { for } \quad 0 \leq i \leq k-1, \\
& h_{k}=a_{k}
\end{aligned}
$$

and

$$
u_{i}=\sum_{j=0}^{i} 1_{A_{j}}, \quad \text { for } \quad 0 \leq i \leq k .
$$

In this case

$$
u^{*}=\sum_{i=0}^{k} h_{i} u_{i}^{*} .
$$

Let us also recall that if $f \in F_{N}$, then there exists

$$
\left\{f_{n}\right\} \subset E_{N} \text { such that } f_{n} \nearrow f \text { and } f_{n}^{*} \nearrow f^{*} \text {. }
$$

Definition 2.1. A function $H:(0, \infty) \times \mathbb{R}_{+}^{m} \longrightarrow \mathbb{R}$ is called an $m$-Carathéodory function when

1) $H\left(\cdot, s_{1}, \ldots, s_{m}\right):(0, \infty) \longrightarrow \mathbb{R}$ is measurable on $(0, \infty) \backslash \Gamma$, where $\Gamma$ is a subset of $(0, \infty)$ having one-dimensional measure zero, for all $s_{1}, \ldots, s_{m} \geq 0$.

2) For all $1 \leq n \leq m$, every $(m-1)$ tuple $s_{i} \geq 0$ and $r \in(0, \infty) \backslash \Gamma$

$$
\begin{aligned}
& \mathbb{R}_{+} \longrightarrow \mathbb{R} \\
& s_{n} \longmapsto H\left(\ldots, s_{n}, \ldots\right)
\end{aligned}
$$

is continuous on $\mathbb{R}_{+}$.

This definition establishes the standard context for handling the measurability of the composite functions involved in (1.1). An important property of an $m$ Carathéodory function is that the composition $x \mapsto H\left(|x|, u_{1}(x), \ldots, u_{m}(x)\right)$ is measurable on $\mathbb{R}^{N}$ for every $u_{1}, \ldots, u_{m} \in F_{N}$.

Definition 2.2. • A function $F: \mathbb{R}_{+}^{2} \longrightarrow \mathbb{R}$ has the property (CZR) when

$$
F(b, d)-F(b, c)-F(a, d)+F(a, c) \geq 0
$$

for all $b \geq a \geq 0$ and $d \geq c \geq 0$. 
- A function $G:(0, \infty) \times \mathbb{R}_{+} \longrightarrow \mathbb{R}$ has the property (CZR-2) when

$$
G(b, d)-G(b, c)-G(a, d)+G(a, c) \leq 0
$$

for all $b \geq a>0$ and $d \geq c \geq 0$.

- A function $H:(0, \infty) \times \mathbb{R}_{+}^{2} \longrightarrow \mathbb{R}$ has the property (CZR-3) when the function $H(\cdot, \cdot, y)-H(\cdot, \cdot, z)$ has the property (CZR-2) for all $y \geq z \geq 0$.

From now on, in an integral where no domain of integration is indicated, it is to be understood that integration extends over all $\mathbb{R}^{N}$.

Lemma 2.1. Let $f, g$ and $h$ be three elements of $F_{N}$. Then

$$
\int f(x) g(x) h(x) d x \leq \int f^{*}(x) g^{*}(x) h^{*}(x) d x .
$$

Proof. We first prove the result for functions in $E_{N}$. Let $f=a 1_{A}, g=b 1_{B}$ and $h=c 1_{C}$ :

$$
\begin{aligned}
\int 1_{A} 1_{B} 1_{C} & =\int 1_{A \cap B \cap C} \leq \min \{\operatorname{mes} A, \operatorname{mes} B, \operatorname{mes} C\} \\
& =\int 1_{A^{*} \cap B^{*} \cap C^{*}}=\int 1_{A^{*}} 1_{B^{*}} 1_{C^{*}} \\
& =\int\left(1_{A}\right)^{*}\left(1_{B}\right)^{*}\left(1_{C}\right)^{*} .
\end{aligned}
$$

Now, let $f, g$ and $h$ be three functions in $E_{N}$ rewritten with respect to (2.2):

$$
f(x)=\sum_{i=0}^{n} a_{i} 1_{A_{i}}(x), \quad g(x)=\sum_{j=0}^{m} b_{j} 1_{B_{j}}(x) \quad \text { and } \quad h(x)=\sum_{k=0}^{\ell} c_{k} 1_{C_{k}}(x) .
$$

Then, by (2.5),

$$
\begin{gathered}
\int f g h=\sum_{i, j, k} a_{i} b_{j} c_{k} \int 1_{A_{i}} 1_{B_{j}} 1_{C_{k}} \leq \sum_{i, j, k} a_{i} b_{j} c_{k} \int\left(1_{A_{i}}\right)^{*}\left(1_{B_{j}}\right)^{*}\left(1_{C_{k}}\right)^{*} \\
=\int f^{*} g^{*} h^{*} \text { by }(2.3) .
\end{gathered}
$$

Using (2.4), we can extend the result to elements of $F_{N}$ thanks to the monotone convergence theorem.

Lemma 2.2 (Generalized Hardy-Littlewood inequality). Let $\left\{f_{i}\right\}_{0 \leq i \leq n}$, where $n \in$ $\mathbb{N}$, be $n$ elements of $F_{N}$. Then

$$
\int \prod_{i=0}^{n} f_{i}(x) d x \leq \int \prod_{i=0}^{n} f_{i}^{*}(x) d x .
$$

\section{MAIN RESUlts}

Theorem 3.1. Let $u, v \in F_{N}$ and $H:(0, \infty) \times \mathbb{R}_{+}^{2} \longrightarrow \mathbb{R}$ be a 2-Carathéodory function verifying:

(H1) $H(r, s, t)=\int_{0}^{t} \int_{0}^{s} \partial_{2} \partial_{3} H(r, a, b) d a d b$ for almost every $r>0$ and every $s, t \geq 0$.

(H2) $\partial_{2} \partial_{3} H(r, s, t) \geq 0$ for every $r>0, s, t \geq 0$. 
(H3) $\partial_{2} \partial_{3} H(\cdot, s, t)$ is decreasing on $(0, \infty)$ for every $s, t \geq 0$. Then

$$
\int H(|x|, u(x), v(x)) d x \leq \int H\left(|x|, u^{*}(x), v^{*}(x)\right) d x .
$$

In addition, we assume:

$$
\int_{0}^{\infty} \int_{0}^{\infty} \int \partial_{2} \partial_{3} H(|x|, s, t) 1_{\left\{u^{*}(x) \geq s\right\}} 1_{\left\{v^{*}(x) \geq t\right\}} d x d t d s<\infty
$$

(H5) (a) $\partial_{2} \partial_{3} H(r, \cdot, t)$ is continuous at zero for almost every $r>0$ and $t \geq 0$.

(b) $\partial_{2} \partial_{3} H(r, s, \cdot)$ is continuous at zero for almost every $r>0$ and $s \geq 0$.

(H6) (a) For any $s \leq 1$, there exist $f_{t}^{1}$ and $f_{t}^{2} \in L^{1}\left(\mathbb{R}^{N}\right)$ such that

$$
\partial_{2} \partial_{3} H(|x|, s, t) 1_{\{u(x) \geq s\}} 1_{\{v(x) \geq t\}} \leq f_{t}^{1}(x)
$$

and

$$
\partial_{2} \partial_{3} H(|x|, s, t) 1_{\left\{u^{*}(x) \geq s\right\}} 1_{\left\{v^{*}(x) \geq t\right\}} \leq f_{t}^{2}(x)
$$

for almost every $x \in \mathbb{R}^{N}$ and $t \geq 0$.

(b) For any $t \leq 1$, there exist $g_{s}^{1}$ and $g_{s}^{2} \in L^{1}\left(\mathbb{R}^{N}\right)$ such that

$$
\partial_{2} \partial_{3} H(|x|, s, t) 1_{\{u(x) \geq s\}} 1_{\{v(x) \geq t\}} \leq g_{s}^{1}(x)
$$

and

$$
\partial_{2} \partial_{3} H(|x|, s, t) 1_{\left\{u^{*}(x) \geq s\right\}} 1_{\left\{v^{*}(x) \geq t\right\}} \leq g_{s}^{2}(x)
$$

for almost every $x \in \mathbb{R}^{N}$ and $s \geq 0$.

(H7) (a) $\partial_{2} \partial_{3} H(\cdot, 0, t)$ is strictly decreasing for almost every $t \geq 0$.

(b) $\partial_{2} \partial_{3} H(\cdot, s, 0)$ is strictly decreasing for almost every $s \geq 0$.

Let $u, v \in F_{N}^{+}$. Then

$$
\int H(|x|, u(x), v(x)) d x=\int H\left(|x|, u^{*}(x), v^{*}(x)\right) d x
$$

if and only if $(u, v)=\left(u^{*}, v^{*}\right)$ almost everywhere.

Proof. Using (H1), we have

$$
\int H(|x|, u(x), v(x)) d x=\iint_{0}^{\infty} \int_{0}^{\infty} \partial_{2} \partial_{3} H(|x|, s, t) 1_{\{s \leq u(x)\}} 1_{\{t \leq v(x)\}} d s d t d x .
$$

(H2) enables us to use Tonelli's theorem. We then can invert the $d s, d t$ and $d x$ integrations in (3.1); thus

$$
\int H(|x|, u(x), v(x)) d x=\int_{0}^{\infty} \int_{0}^{\infty} \int \partial_{2} \partial_{3} H(|x|, s, t) 1_{\{u(x) \geq s\}} 1_{\{v(x) \geq t\}} d x d s d t .
$$


(H3) ensures that $\partial_{2} \partial_{3} H(|x|, s, t)$ is radially decreasing for any $s, t \geq 0$. For $s, t \geq 0$, set $\ell_{s, t}=\lim _{|x| \rightarrow \infty} \partial_{2} \partial_{3} H(|x|, s, t)$. Then

$$
\begin{aligned}
& \int \partial_{2} \partial_{3} H(|x|, s, t) 1_{\{u(x) \geq s\}} 1_{\{v(x) \geq t\}} d x \\
& =\int\left\{\partial_{2} \partial_{3} H(|x|, s, t)-\ell_{s, t}\right\} 1_{\{u(x) \geq s\}} 1_{\{v(x) \geq t\}} d x \\
& \quad+\int \ell_{s, t} 1_{\{u(x) \geq s\}} 1_{\{v(x) \geq t\}} d x .
\end{aligned}
$$

(H3) implies that, for all $s, t \geq 0$,

$$
\left(\partial_{2} \partial_{3} H(|x|, s, t)-\ell_{s, t}\right)^{*}=\partial_{2} \partial_{3} H(|x|, s, t)-\ell_{s, t} \quad \text { for almost every } x \in \mathbb{R}^{N} .
$$

Thus, by Lemma 2.1,

$$
\begin{aligned}
& \int\left\{\partial_{2} \partial_{3} H(|x|, s, t)-\ell_{s, t}\right\} 1_{\{u(x) \geq s\}} 1_{\{v(x) \geq t\}} d x \\
\leq & \int\left\{\partial_{2} \partial_{3} H(|x|, s, t)-\ell_{s, t}\right\} 1_{\left\{u^{*}(x) \geq s\right\}} 1_{\left\{v^{*}(x) \geq t\right\}} d x
\end{aligned}
$$

and since $\ell_{s, t} \geq 0$, we certainly have

$$
\int \ell_{s, t} 1_{\{u(x) \geq s\}} 1_{\{v(x) \geq t\}} d x \leq \int \ell_{s, t} 1_{\left\{u^{*}(x) \geq s\right\}} 1_{\left\{v^{*}(x) \geq t\right\}} d x .
$$

Combining (3.3) to (3.5), we obtain for all $s, t \geq 0$,

$$
\begin{aligned}
& \int \partial_{2} \partial_{3} H(|x|, s, t) 1_{\{u(x) \geq s\}} 1_{\{v(x) \geq t\}} d x \\
\leq & \int \partial_{2} \partial_{3} H(|x|, s, t) 1_{\left\{u^{*}(x) \geq s\right\}} 1_{\left\{v^{*}(x) \geq t\right\}} d x .
\end{aligned}
$$

Clearly (3.2) holds when $u$ is replaced by $u^{*}$ and $v$ by $v^{*}$. Thus (3.2) together with (3.6) imply that

$$
\int H(|x|, u(x), v(x)) d x \leq \int H\left(|x|, u^{*}(x), v^{*}(x)\right) d x,
$$

proving the first part of our result.

Now suppose that

$$
\int H(|x|, u(x), v(x)) d x=\int H\left(|x|, u^{*}(x), v^{*}(x)\right) d x .
$$

That is to say,

$$
\begin{aligned}
& \int_{0}^{\infty} \int_{0}^{\infty} \int \partial_{2} \partial_{3} H(|x|, s, t) 1_{\{u(x) \geq s\}} 1_{\{v(x) \geq t\}} d x d s d t \\
= & \int_{0}^{\infty} \int_{0}^{\infty} \int \partial_{2} \partial_{3} H(|x|, s, t) 1_{\left\{u^{*}(x) \geq s\right\}} 1_{\left\{v^{*}(x) \geq t\right\}} d x d s d t .
\end{aligned}
$$


Then (H4) and (3.6) imply that equality occurs in the previous line if and only if: For almost every $(s, t) \in \mathbb{R}_{+}^{2}$,

$$
\begin{gathered}
\int \partial_{2} \partial_{3} H(|x|, s, t) 1_{\{u(x) \geq s\}} 1_{\{v(x) \geq t\}} d x \\
=\int \partial_{2} \partial_{3} H(|x|, s, t) 1_{\left\{u^{*}(x) \geq s\right\}} 1_{\left\{v^{*}(x) \geq t\right\}} d x(<\infty) .
\end{gathered}
$$

(3.8) may not hold for $D=\{(s, 0) \mid s \geq 0\}$, however we can certainly construct a sequence $\left\{t_{n}\right\} \subset \mathbb{R}_{+}$such that $t_{n} \searrow 0$ and (3.8) occurs for $D_{n}=\left\{\left(s, t_{n}\right) \mid s \geq 0\right\}$ for all $n \in \mathbb{N}$.

Thanks to (H5)(b) and (H6)(b), we can use the dominated convergence theorem obtaining: for almost every $s \geq 0$,

$$
\int \partial_{2} \partial_{3} H(|x|, s, 0) 1_{\{u(x) \geq s\}} d x=\int \partial_{2} \partial_{3} H(|x|, s, 0) 1_{\left\{u^{*}(x) \geq s\right\}} d x<\infty .
$$

Similarly, for almost every $t \geq 0$,

$$
\int \partial_{2} \partial_{3} H(|x|, 0, t) 1_{\{v(x) \geq t\}} d x=\int \partial_{2} \partial_{3} H(|x|, 0, t) 1_{\left\{v^{*}(x) \geq t\right\}} d x<\infty .
$$

Finally (H7) enables us to use Lemma 3.6 of [1], concluding that (3.9) occurs if and only if $u=u^{*}$ a.e. and (3.10) holds if and only if $v=v^{*}$ a.e.

Example 3.1. $H(r, s, t)=\frac{1}{1+r^{2}} s t, u, v \in F_{1}^{+}$such that

$$
\int \frac{1}{1+|x|^{2}} u^{*}(x) v^{*}(x) d x<\infty
$$

satisfy all the hypotheses of Theorem 3.1.

Remarks 3.1. i) In (H1), it is not required that $\partial_{2} \partial_{3} H$ exists everywhere. The class of functions satisfying (H1) are discussed in detail in [4.

ii) a) If (H1) only holds for $s \in \operatorname{Im} u \cup \operatorname{Im} u^{*}$ and $t \in \operatorname{Im} v \cup \operatorname{Im} v^{*}$, then Theorem 3.1 remains true.

b) If (H2) and (H3) hold for almost every $s \in \operatorname{Im} u \cup \operatorname{Im} u^{*}$ and almost every $t \in \operatorname{Im} v \cup \operatorname{Im} v^{*}$, then the conclusion of Theorem 3.1 remains valid.

iii) In (H5), we can replace the continuity of the functions by the existence of

$$
\lim _{s \rightarrow 0^{+}} \partial_{2} \partial_{3} H(r, s, t) \text { and } \lim _{t \rightarrow 0^{+}} \partial_{2} \partial_{3} H(r, s, t),
$$

provided we modify $(\mathrm{H} 7)$.

iv) If $H \in C^{3}\left((0, \infty) \times \mathbb{R}_{+}^{2}\right)$,

(H3) $\Longleftrightarrow \partial_{1} \partial_{2} \partial_{3} H(r, s, t) \leq 0$

$(\mathrm{H} 7)(\mathrm{a}) \Longleftrightarrow \partial_{1} \partial_{2} \partial_{3} H(r, 0, t)<0$,

for all $r>0, s, t \geq 0$,

$(\mathrm{H} 7)(\mathrm{b}) \Longleftrightarrow \partial_{1} \partial_{2} \partial_{3} H(r, s, 0)<0$,

for all $r>0$, almost every $t \geq 0$,

for all $r>0$, almost every $s \geq 0$.

Remark 3.2. If (H1), (H2), (H3), (H4) and (a) of (H5), (H6) and (H7) hold, then

$$
\int H(|x|, u(x), v(x)) d x=\int H\left(|x|, u^{*}(x), v^{*}(x)\right) d x
$$

implies that $v=v^{*}$ a.e. 
Remark 3.3. A priori Theorem 3.1 does not apply to such functions as

$$
H(r, s, t)=\frac{1}{1+r^{2}} s^{n} t^{m}, \quad n, m>1 .
$$

However, by a suitable change of integrands, we can determine cases of equality for such functions using this result. More precisely, suppose that:

*) $\quad \int \frac{1}{1+|x|^{2}} f^{n}(x) g^{m}(x) d x=\int \frac{1}{1+|x|^{2}}\left(f^{*}(x)\right)^{n}\left(g^{*}(x)\right)^{m} d x<\infty$, where $f$ and $g \in F_{1}^{+}$.

Set $F=f^{n}$. Then $F \in F_{1}^{+}$and $F^{*}=\left(f^{n}\right)^{*}=\left(f^{*}\right)^{n}$ a.e. and $G=g^{m} \in F_{1}^{+}$ with $G^{*}=\left(g^{m}\right)^{*}=\left(g^{*}\right)^{m}$ a.e.

*) is equivalent to $\int \frac{1}{1+|x|^{2}} F(x) G(x) d x=\int \frac{1}{1+|x|^{2}} F^{*}(x) G^{*}(x) d x<\infty$.

Applying Example 3.1, we conclude that $F=F^{*}$ a.e. and $G=G^{*}$ a.e., thus $(f, g)=\left(f^{*}, g^{*}\right)$ a.e.

Theorem 3.1 can be easily generalized to $m$ functions.

Theorem 3.2. Let $u_{1}, \ldots, u_{m} \in F_{N}, H:(0, \infty) \times \mathbb{R}_{+}^{m} \longrightarrow \mathbb{R}$ be an $m$-Carathéodory function such that:

i) $H\left(r, b_{1}, \ldots, b_{m}\right)=\int_{0}^{b_{m}} \ldots \int_{0}^{b_{1}} \partial_{2} \ldots \partial_{m+1} H\left(r, a_{1}, \ldots, a_{m}\right) d a_{1} \ldots d a_{m}$ for all $r>0, b_{1}, \ldots, b_{m} \geq 0$.

ii) $\partial_{2} \ldots \partial_{m+1} H\left(r, a_{1}, \ldots, a_{m}\right) \geq 0$ for all $r>0, a_{1}, \ldots, a_{m} \geq 0$.

iii) $r \mapsto \partial_{2} \ldots \partial_{m+1} H\left(r, a_{1}, \ldots, a_{m}\right)$ is non-increasing for all $a_{1}, \ldots, a_{m}$ $\geq 0$. Then

$$
\int H\left(|x|, u_{1}(x), \ldots, u_{m}(x)\right) d x \leq \int H\left(|x|, u_{1}^{*}(x), \ldots, u_{m}^{*}(x)\right) d x
$$

We suppose in addition that:

iv) $\int_{\mathbb{R}_{+}^{m}} \int \partial_{2} \ldots \partial_{m+1} H\left(|x|, a_{1}, \ldots, a_{m}\right) 1_{\left\{u_{1}^{*}(x) \geq a_{1}\right\}} \ldots 1_{\left\{u_{m}^{*}(x) \geq a_{m}\right\}} d x d a_{1} \ldots d a_{m}$ $<\infty$.

v) For any fixed $n, 1 \leq n \leq m, \partial_{2} \ldots \partial_{m+1} H\left(r, a_{n}, \cdot\right)$ is continuous at $0_{\mathbb{R}^{m-1}}$ for all $r>0$ and $a_{n} \geq 0$.

vi) There exists an integrable function $K: \mathbb{R}^{N} \longrightarrow \mathbb{R}_{+}$such that

$$
\partial_{2} \ldots \partial_{m+1} H\left(|x|, a_{1}, \ldots, a_{m}\right) \leq K(x) \quad \text { for all } x \in \mathbb{R}^{N}, a_{1}, \ldots, a_{m} \geq 0 \text {. }
$$

vii) For all fixed $n, 1 \leq n \leq m$, the function $r \mapsto \partial_{2} \ldots \partial_{m+1} H\left(r, a_{n}, 0_{\mathbb{R}^{m-1}}\right)$ is strictly decreasing on $(0, \infty)$ for all $a_{n} \geq 0$.

Let $u_{i} \in F_{N}^{+}$. Then

$$
\int H\left(|x|, u_{1}(x), \ldots, u_{m}(x)\right) d x=\int H\left(|x|, u_{1}^{*}(x), \ldots, u_{m}^{*}(x)\right) d x
$$

if only if each $u_{i}$ is Schwarz symmetric.

Remark 3.4. vi) can be expressed in a same manner as (H6) which is important for applications of the previous result in the calculus of variations.

All the hypotheses can be weakened in a same manner as in Remarks 3.1.

Theorem 3.3. Let $u, v \in F_{N}, H:(0, \infty) \times \mathbb{R}_{+}^{2} \longrightarrow \mathbb{R}$ be a 2-Carathéodory function satisfying:

1) $H(r, \cdot, \cdot)$ has the property (CZR) for almost every $r>0$,

2) $H$ has the property (CZR-3). 
3) (a) For almost every $r>0$ and all $t \geq 0$,

$$
H(r, 0, t)-H(r, 0,0)=\int_{0}^{t} \partial_{3} H(r, 0, b) d b .
$$

(b) For almost every $r>0$ and all $s \geq 0$,

$$
H(r, s, 0)-H(r, 0,0)=\int_{0}^{s} \partial_{2} H(r, a, 0) d a .
$$

4) (a) $\int_{0}^{\infty} \int\left|\partial_{3} H(|x|, 0, t)\right| 1_{\{v(x) \geq t\}} d x d t$ and $\int_{0}^{\infty} \int\left|\partial_{3} H(|x|, 0, t)\right| 1_{\left\{v^{*}(x) \geq t\right\}} d x d t$ are finite.

(b) $\int_{0}^{\infty} \int\left|\partial_{2} H(|x|, s, 0)\right| 1_{\{u(x) \geq s\}} d x d s$ and $\int_{0}^{\infty} \int\left|\partial_{2} H(|x|, s, 0)\right| 1_{\left\{u^{*}(x) \geq s\right\}} d x d s$ are finite.

5) (a) $r \mapsto \partial_{3} H(r, 0, t)$ is strictly decreasing on $(0, \infty)$ for almost every $t \geq$ 0 .

(b) $r \mapsto \partial_{2} H(r, s, 0)$ is strictly decreasing on $(0, \infty)$ for almost every $s \geq 0$.

6) $\int H(|x|, 0,0) d x<\infty$.

Then if $\int H\left(|x|, u^{*}(x), v^{*}(x)\right) d x<\infty$,

$$
\int H(|x|, u(x), v(x)) d x=\int H\left(|x|, u^{*}(x), v^{*}(x)\right) d x
$$

if and only if $(u, v)=\left(u^{*}, v^{*}\right)$ a.e.

Proof. For $r>0, s, t \geq 0$, set

$$
\tilde{H}(r, s, t)=H(r, s, t)-H(r, s, 0)-H(r, 0, t)+H(r, 0,0) .
$$

1) and 2) imply that $\tilde{H}$ satisfies all the hypotheses of Proposition 4.1 of [2]. Then for any $u, v \in F_{N}$,

$$
\int \tilde{H}(|x|, u(x), v(x)) d x \leq \int \tilde{H}\left(|x|, u^{*}(x), v^{*}(x)\right) d x .
$$

We can easily check using 3$) \rightarrow 6$ ) that

$$
\begin{aligned}
& -\infty<\int H(|x|, u(x), 0) d x \leq \int H\left(|x|, u^{*}(x), 0\right) d x<\infty, \\
& -\infty<\int H(|x|, 0, v(x)) d x \leq \int H\left(|x|, 0, v^{*}(x)\right) d x<\infty .
\end{aligned}
$$

Now suppose that

$$
\int H(|x|, u(x), v(x)) d x=\int H\left(|x|, u^{*}(x), v^{*}(x)\right) d x .
$$

Let $H_{-}(r, s, t)=\max \{-H(r, s, t), 0\}$. Then

$$
\int H_{-}(|x|, u(x), v(x)) d x \quad \text { and } \quad \int H_{-}\left(|x|, u^{*}(x), v^{*}(x)\right) d x
$$


are finite (see proof of Theorem 4.2 of [2]), and we certainly have by Lemma 3.1 of [2] that

$$
\begin{aligned}
\int H(|x|, u(x), v(x)) d x= & \int \tilde{H}(|x|, u(x), v(x)) d x+\int H(|x|, 0, v(x)) d x \\
& +\int H(|x|, u(x), 0) d x-\int H(|x|, 0,0) d x
\end{aligned}
$$

and

$$
\begin{aligned}
\int H\left(|x|, u^{*}(x), v^{*}(x)\right) d x= & \int \tilde{H}\left(|x|, u^{*}(x), v^{*}(x)\right) d x+\int H\left(|x|, 0, v^{*}(x)\right) d x \\
& +\int H\left(|x|, u^{*}(x), 0\right) d x-\int H(|x|, 0,0) d x .
\end{aligned}
$$

Combining (3.12) to (3.15), it follows, since by our hypotheses integrals in (3.12) are finite,

$$
\begin{aligned}
\int \tilde{H}(|x|, u(x), v(x)) d x & =\int \tilde{H}\left(|x|, u^{*}(x), v^{*}(x)\right) d x, \\
\int H(|x|, u(x), 0) d x & =\int H\left(|x|, u^{*}(x), 0\right) d x \\
\int H(|x|, 0, v(x)) d x & =\int H\left(|x|, 0, v^{*}(x)\right) d x .
\end{aligned}
$$

$3) \rightarrow 6$ ) imply, using Theorem 6.1 of [1], that (3.17) and (3.18) hold if and only if $(u, v)=\left(u^{*}, v^{*}\right)$ a.e.

Remark 3.5. Note that 2) and 5)(a) imply that $r \mapsto \partial_{3} H(r, s, t)$ is strictly decreasing for any $s \geq 0$ and almost every $t \geq 0$.

Example 3.2. $H(r, s, t)=\frac{1}{1+r^{2}} s+\frac{1}{1+r^{2}} t, u$ and $v \in F_{1}$ such that

$$
\int \frac{1}{1+|x|^{2}} u^{*}(x) d x \text { and } \int \frac{1}{1+|x|^{2}} v^{*}(x) d x<\infty
$$

satisfy all the hypotheses of Theorem 3.3.

Clearly $\frac{1}{1+r^{2}} s+\frac{1}{1+r^{2}} t$ does not satisfy conditions of Theorem 3.1. Conversly the function given in Example 3.1 does not satisfy the assumptions of Theorem 3.3.

Remark 3.6. In Theorem 3.1, it is assumed that $H(r, \cdot, 0) \equiv H(r, 0, \cdot)=0$. In Theorem 3.3, we suppose that $H$ does not vanish at the hyperplanes; however our approach remains fruitful even though $H(r, \cdot, 0)=0$ and $H(r, 0, \cdot) \neq 0$. More precisely, suppose that

$$
H(r, s, t)=\int_{0}^{t} \int_{0}^{s} \partial_{2} \partial_{3} H(r, a, b) d a d b+H(r, s, 0)+H(r, 0, t)-H(r, 0,0)
$$

for almost every $r>0$, every $s \in \operatorname{Im} u \cup \operatorname{Im} u^{*}$ and every $t \in \operatorname{Im} v \cup \operatorname{Im} v^{*}$.

Closely following the proof of Theorem 3.3, we can easily determine hypotheses on $H$ such that (3.18) holds, and we conclude that $v=v^{*}$ a.e. The establishment of cases of equality in (1.1) is then reduced to $\int H\left(|x|, u(x), v^{*}(x)\right) d x=$ $\int H\left(|x|, u^{*}(x), v^{*}(x)\right) d x$.

Considering

$$
\begin{aligned}
G:(0, \infty) \times \mathbb{R}_{+} & \longrightarrow \mathbb{R}, \\
(r, s) & \longmapsto H(r, s, h(r)),
\end{aligned}
$$


where $h:(0, \infty) \rightarrow \mathbb{R}$ is defined by $h(|x|)=v^{*}(x)$ for every $x \in \mathbb{R}^{N}$ and applying Theorem 6.1 of [1] to $(G, u)$, we determine hypotheses such that $u=u^{*}$ a.e.

Finally, let us point out that a combination of Theorem 3.1 and Theorem 6.1 of [1] also gives appropriate conditions on $H$ such that equality holds in (1.1) if and only if $(u, v)=\left(u^{*}, v^{*}\right)$ a.e.

\section{Some Applications}

For $u=\left(u_{1}, u_{2}\right) \in\left(H^{1}\left(\mathbb{R}^{N}\right)\right)^{2}$, we set

$$
\begin{aligned}
\tilde{J}(u)=\tilde{J}\left(u_{1}, u_{2}\right)= & \frac{1}{2} \int\left|\nabla u_{1}\right|^{2}+\frac{1}{2} \int\left|\nabla u_{2}\right|^{2}-\frac{1}{2} \int p(|x|)|u(x)|^{2} d x \\
& -\int H\left(|x|, u_{1}(x), u_{2}(x)\right) d x .
\end{aligned}
$$

$\left(\tilde{P}_{c}\right): \tilde{I}_{c}=\inf _{u \in \tilde{S}_{c}} \tilde{J}(u)$, where $\tilde{S}_{c}=\left\{u \in\left(H^{1}\left(\mathbb{R}^{N}\right)\right)^{2} \mid\|u\|_{2}^{2}=c^{2}\right\}$ for a prescribed $c>0$.

$\|\cdot\|_{2}$ is the norm in $\left(L^{2}\left(\mathbb{R}^{N}\right)\right)^{2}$. Closely following the proof of Example 5 of [3] whose principal ingredient is (1.1) with $m=1$, we obtain:

Theorem 4.1. Suppose that $p:(0, \infty) \longrightarrow \mathbb{R}_{+}$satisfies:

(P1) $p$ is non-increasing and $\lim _{r \rightarrow \infty} p(r)=0$.

(P2) - If $N=1,2$, there exists $a>0$ such that $p(a)>0$.

- If $N>2$, there exists $R>0$ such that

$$
p(R)>\frac{j_{N / 2-1,1}^{2}}{R^{2}},
$$

where $j_{N / 2-1,1}$ is the first zero of the Bessel function $J_{N / 2-1}$.

Assume that $H:(0, \infty) \times \mathbb{R}^{2} \longrightarrow \mathbb{R}$ is a 2-Carathéodory function such that:

(V1) $H\left(r, s_{1}, s_{2}\right) \leq H\left(r,\left|s_{1}\right|,\left|s_{2}\right|\right)$ for almost every $r>0$ and all $s_{1}, s_{2} \in \mathbb{R}$.

For $r>0, s_{1}$ and $s_{2} \geq 0$, set $s=\left(s_{1}, s_{2}\right)$ and suppose:

(V2) $0 \leq \tilde{H}\left(r, s_{1}, s_{2}\right) \leq K\left(|s|^{2}+\sum_{i=1}^{2} s_{i}^{\ell+2}\right)$, where $K>0,0<\ell<\frac{4}{N}$, and $\tilde{H}$ is the restriction of $H$ to $(0, \infty) \times \mathbb{R}_{+}^{2}$.

(V3) (a) $\left(r, s_{1}\right) \mapsto \tilde{H}\left(r, s_{1}, s_{2}\right)$ and $\left(r, s_{2}\right) \mapsto \tilde{H}\left(r, s_{1}, s_{2}\right)$ have the property (CZR-2) for all $s_{2} \geq 0$ (respectively $s_{1} \geq 0$ ),

(b) $\tilde{H}$ has the property (CZR) for almost every $r>0$,

(c) $\tilde{H}$ has the property (CZR-3),

d) $s_{1} \mapsto \lim _{r \rightarrow \infty} \tilde{H}\left(r, s_{1}, 0\right)$ and $s_{2} \mapsto \lim _{r \rightarrow \infty} \tilde{H}\left(r, 0, s_{2}\right)$ exist and are continuous $n[0, \infty)$.

(V4) $\forall \varepsilon>0, \exists r_{0}>0$ and $s_{0}>0$ such that

$$
\tilde{H}\left(r, s_{1}, s_{2}\right) \leq \varepsilon|s|^{2} \quad \text { for all } r \geq r_{0} \text { and }|s| \leq s_{0} .
$$

(V5) $\tilde{H}\left(r, \theta s_{1}, \theta s_{2}\right) \geq \theta^{2} \tilde{H}\left(r, s_{1}, s_{2}\right)$ for almost every $r>0$, every $\theta \geq 1$ and $s_{1}, s_{2} \geq 0$.

Then for any $c>0$, there exists $u^{c}=\left(u_{1}^{c}, u_{2}^{c}\right)$ such that each component is Schwarz symmetric, $\left\|u^{c}\right\|_{2}^{2}=c^{2}$ and $\tilde{J}\left(u^{c}\right)=\tilde{I}_{c}$. 
Theorem 4.2. Suppose that $(p, H)$ satisfy conditions of Theorem 4.1. If in addition $p$ is strictly decreasing on $(0, \infty)$ or $H$ verifies 3$) \rightarrow 5)$ of Theorem 3.3 , then all minimizers of $\left(\tilde{P}_{c}\right)$ are Schwarz symmetric. (This means that all the minimizers of $\left(\tilde{P}_{c}\right)$ can be written $\left(u_{1}^{c}, u_{2}^{c}\right)$, where $u_{1}^{c}$ and $u_{2}^{c}$ are Schwarz symmetric.)

Remark 4.1. Under appropriate growth conditions on $H$ (see Theorem 6.1 (CV) of [1]), 4) of Theorem 3.3 is satisfied for every $u, v \in H^{1}\left(\mathbb{R}^{N}\right)$.

Remark 4.2. Under some regularity assumptions on $H$, a solution of $\left(\tilde{P}_{c}\right)$ satisfies the $2 \times 2$ elliptic eigenvalue system

$$
S_{2 \times 2}: \quad\left\{\begin{array}{l}
\Delta u(x)+\partial_{2} H(|x|, u(x), v(x))+p(|x|) u(x)+\lambda u(x)=0, \\
\Delta v(x)+\partial_{3} H(|x|, u(x), v(x))+p(|x|) v(x)+\lambda v(x)=0,
\end{array}\right.
$$

where $x \in \mathbb{R}^{N}$ and $\lambda \in \mathbb{R}$ is a Lagrange multiplier.

Theorem 4.1 assures us that $S_{2 \times 2}$ admits a Schwarz symmetric ground state (that is to say, each component of the ground state is Schwarz symmetric).

Under conditions of Theorem 4.2, all ground states of $S_{2 \times 2}$ are Schwarz symmetric.

\section{REFERENCES}

[1] Hajaiej H., Cases of Equality and Strict Inequality in the Extended Hardy-Littlewood Inequalities, Proc. Roy. Soc. Edinburgh, 135A (2005), 643-661.

[2] Hajaiej H., Stuart C. A., Extensions of the Hardy-Littlewood Inequalities for Schwarz Symmetrization, Int. J. Math. Math. Sci. 2004, 3129-3150. MR2110793

[3] Hajaiej H., Stuart C. A., Existence and Non-existence of Schwarz Symmetric Ground States for Elliptic Eigenvalue Problems, Matematica pura ed Applicata, 186, electronic, 2004.

[4] Folland G.B., Real Analysis: Modern Techniques and their Applications, Pure and Applied Mathematics, John Wiley and Sons, New York, 1999. MR.1681462 (2000c:00001)

[5] Tahraoui R., Symmetrization Inequalities, Nonlinear Analysis TMA, 27 (1996), pp. 933-955, Corrigendum 33 (2000), 535. MR1404592(97h:35008) MR:1725394

Department of Mathematics, University of Virginia, Charlottesville, Virginia 229044137 